\title{
Imaging Quiz
}

\author{
J. Devkota, M.D., \\ Columbia, Missouri
}

DOI: http://dx.doi.org/10.5915/20-3-13288

\section{Imaging Quiz}

A 42-year-old female complained of intermittent right flank pain and mild swelling of her feet for a month. She noticed blood in the urine and came to the hospital for an IVP, CT scan of the abdomen, and then an arteriogram. She had been perfectly healthy for the six months prior to the admission. She denied weight loss, loss of appetite, easy fatiguability or fever. Physical examination showed only costovertebral angle tenderness and right flank fullness. During the hospitalization, the patient became anuric. Laboratory tests showed mild polycythemia and red blood cells in the urine.

Here are a few of this patient's radiographs. What is your working diagnosis? How would you proceed in the management of this patient if your hospital had the following diagnostic and therapeutic capabilities:

1. Angiography

2. Urine analysis and blood tests

3. KUB

4. IVP

5. Ultrasound

6. GI Series and BE

7. CT Scan

8. Radionuclide Renal Scan

9. Inferior Venacavagram

10. Retrograde Pyelogram

11. Embolization

12. Embolysis Therapy

13. Radiation Therapy only

14. Radical Nephrectomy

15. Simple Nephrectomy and Radiation

16. Chemotherapy

17. Do nothing because it's hopeless

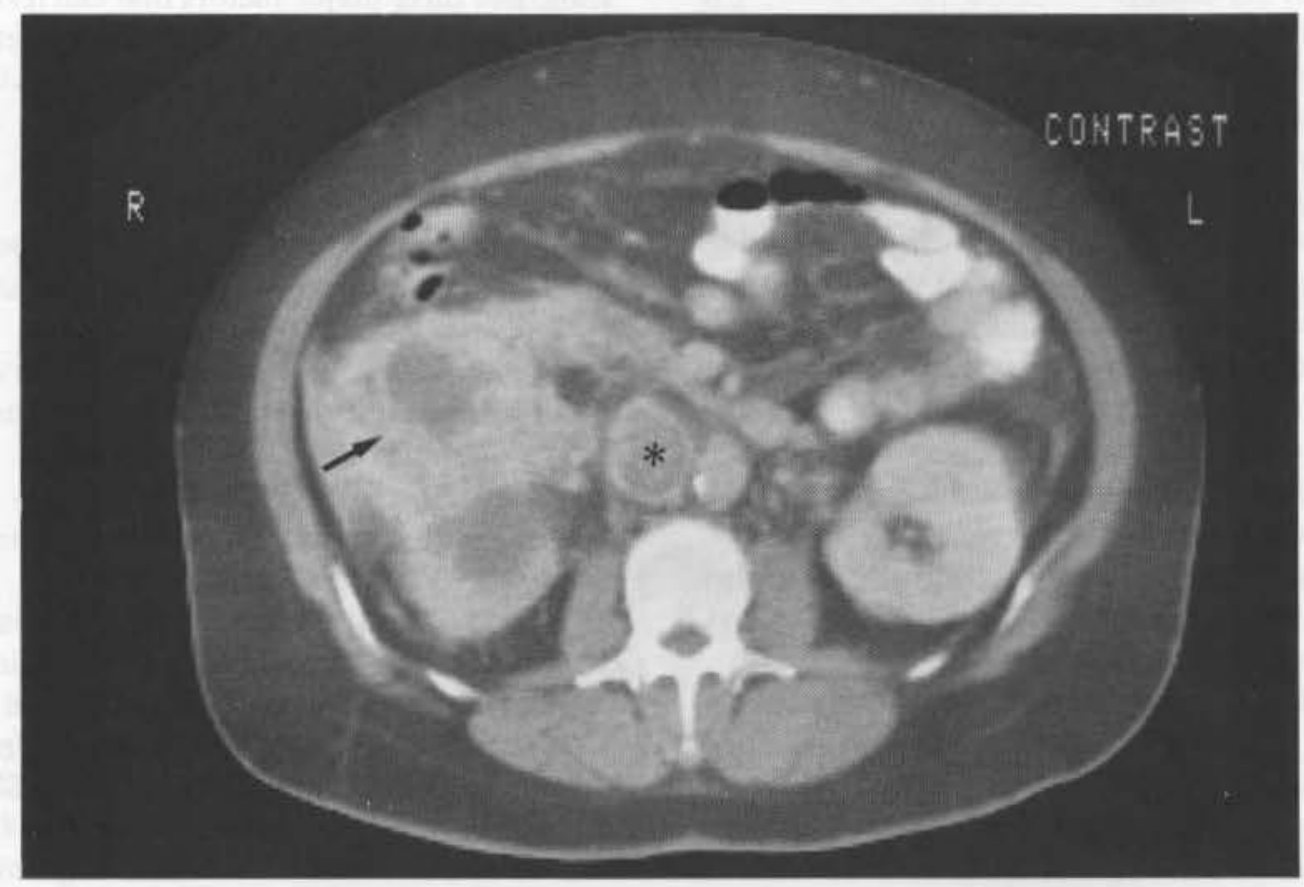

Figure 1

From the Department of Radiology

University of Missouri-Columbia

Reprint requests: J. Devkota, M.D.

Department of Radiology Room M-201

University of Missouri-Columbia Columbia, MO 65212 


\section{Diagnosis}

This case represents aggressive right renal cell carcinoma with extension to the inferior vena-cava and both renal veins

One logical sequence of management would be the rapeutic options numbered: $2,3,4,5$ or $6,8,1,9$, $11,15,13$ or 16 .

If a renal tunor is suspected, and IVP, CT, and angiograms are essential tests and preoperative tumor embolization is a desirable procedure.

This patient's CT scan shows a large necrotic tumor mass (arrow) with invasion into the IVC and renal veins (Fig. 1). The inferior venacavagram shows complete occlusion of the IVC and renal veins (arrow) which explains the pedal edema and anuria (Fig. 2). Since the tumor appeared very vascular on arteriogram (Fig. 3), it was decided to embolize the large feeding arteries (white arrow) to minimize the operative blood loss and enhance dissection of the mass during surgery (Fig. 4). Since the tumor had invaded the vascular structures, a palliative simple nephrectomy followed by local radiation therapy and systemic chemotherapy were the preferred treatments.

Renal cell carcinoma is the most common malignant tumor of the kidney, comprising about $85-90 \%$ of all renal tumors. It is of renal tubular epithelial cell origin. ${ }^{1}$ The etiology of this carcinoma is unknown but it is considered to be induced by chemical and viral agents. An increased incidence has been seen in smokers. The-male-to-female ratio is $3: 1 .^{1,2}$

The textbook triad of hematuria, flank pain and an abdominal mass is only seen in $10 \%$ of patients. ${ }^{1-6}$ Microscopic hematguria is the most common finding. The tumor is usually very large. Local invasion and distant metastasis via the inferior vena-cava and the renal veins are common. Metastasis has been reported up to twenty years post nephrectomy. ${ }^{2-7}$

\section{References}

1. Teplick and Haskim: Roentgenologic Diagnosis. Philadelphia, W.B. Saunders, 1976;852-56.

2. Cronan JJ, Zeman RK, Rosenfield AT: Comparison of computerized tomography, ultrasound and angiography in staging renal cell carcinoma. J Urol, 1982;127:712-14.

3. Nemoto R, Kato T, Iwata K, et al: Evaluation of therapeutic arterial embolization in renal cell carcinoma using microencapsulated mitomycin C. Radiology, 1981;141:572.

4. Gildiner M, Samu PC: Arteriographic demonstration of extension of renal carcinoma into renal vein and vena-cava. Radiology, 1980;136:578.

5. Wallace S, Chuang VP, Swanson DA, Bracken B, et al. Embolization of renal carcinoma: Experience with 100 patients. Radiology, 1981;138:563.

6. Madayag MA, Ambos MA, LeFleur RS, Bosniak MA: Involvement of the inferior vena-cava in patients with renal cell carcinoma. Radiology, 1979;133:321-26.

7. deKernion JB, Berry D: Diagnosis and treatment of renal cell carcinoma. Cancer, 1980;45:1947-56. 